United Nations Educational Scientific and Cultural Organization

and

International Atomic Energy Agency

THE ABDUS SALAM INTERNATIONAL CENTRE FOR THEORETICAL PHYSICS

\title{
INTEGRABILITY AND SOLITON IN A CLASSICAL ONE DIMENSIONAL SITE DEPENDENT BIQUADRATIC HEISENBERG SPIN CHAIN AND THE EFFECT OF NONLINEAR INHOMOGENEITY
}

\author{
L. Kavitha ${ }^{1}$ \\ Department of Physics, Lady Doak College, Madurai - 625 002, India \\ and \\ M. Daniel ${ }^{2}$ \\ Centre for Nonlinear Dynamics, Department of Physics, Bharathidasan University, \\ Tiruchirapalli - 620 024, India \\ and \\ The Abdus Salam International Centre for Theoretical Physics, Trieste, Italy.
}

\begin{abstract}
The integrability of one dimensional classical continuum inhomogeneous biquadratic Heisenberg spin chain and the effect of nonlinear inhomogeneity on the soliton of an underlying completely integrable spin model are studied. The dynamics of the spin system is expressed in terms of a higher order generalized nonlinear Schrödinger equation through a differential geometric approach which becomes integrable for a particular choice of the biquadratic exchange interaction and for linear inhomogeneity. The effect of nonlinear inhomogeneity on the spin soliton is studied by carrying out a multiple scale perturbation analysis.

\section{MIRAMARE - TRIESTE}

July 2002

\footnotetext{
1 kavithalouis@yahoo.com

${ }^{2}$ Regular Associate of the Abdus Salam ICTP. Corresponding author: muthiahdaniel@yahoo.com
} 


\section{INTRODUCTION}

One dimensional classical continuum Heisenberg ferromagnetic spin chains with different magnetic interactions act as an interesting class of nonlinear dynamical systems exhibiting a rich variety of integrability

properties and soliton spin excitations. Though the dynamics of these spin systems are governed by Landau-Lifshitz(LL) equation[1], a highly nontrivial vector nonlinear partial differential equation, the space curve mapping procedure developed by Lakshmanan $[2,3]$ and the gauge equivalence method proposed by Zakharov and Takhtajan [4] which were originally used in the case of one dimensional classical isotropic Heisenberg ferromagnetic spin chain were considered to be useful approaches that help to map the LL equation to nonlinear Schrödinger(NLS) family of equations. The geometric equivalence method under space curve mapping was then used by Lakshmanan and his collaborators for several ferromagnetic spin systems with nontrivial and higher order magnetic interactions as well as for some mathematical generalizations of the LL equation and several integrable spin models identified and the underlying soliton spin excitations explored[57]. The classical Heisenberg ferromagnetic spin chains with biquadratic interaction, Dzyaloshinski - Moriya interaction and octupole-dipole interaction are some of the physically interesting spin models, the nonlinear dynamics of which have been understood and integrable models identified in recent years[5-10]. In this context, the study of classical isotropic Heisenberg spin chain[3] and site dependent isotropic bilinear spin chain $[11,12]$ are considered to be pioneering nonlinear spin models for they were modeled by the completely integrable cubic NLS equation and an inhomogeneous NLS equation which were considered to be important completely integrable nonlinear evolution equations in their own respect[11-13]. In this paper, we study the nonlinear spin dynamics of a one dimensional classical isotropic biquadratic Heisenberg spin chain by introducing varying bilinear and biquadratic exchange interactions along the spin lattice. We try to identify the underlying completely integrable spin models and construct soliton spin excitations. In the nonintegrable case, we carry out a multiple scale perturbation analysis to understand the effect of nonlinear inhomogeneity on the spin soliton and also the perturbed soliton constructed.

The plan of the paper is as follows. In Section II, we present the model and dynamical equation for the spin system and map the continuous spin chain onto a moving helical space curve and express the dynamics in terms of the evolution of the curvature and torsion of the space curve which is found to be equivalent to a generalized inhomogeneous higher order NLS equation in Section III. In order to see whether the above equation is integrable or not we carry out Painlevé singularity structure analysis in Section IV and identify the underlying integrable spin models. After mentioning the integrability properties we carry out a perturbation analysis to construct perturbed soliton and to study the effect of nonlinear inhomogeneity in Section V. The results are concluded in Section VI. 


\section{MODEL AND CLASSICAL EQUATION OF MOTION}

The Heisenberg Hamiltonian for a one dimensional classical ferromagnetic spin chain with $\mathrm{N}$ spins interacting with their nearest neighbours and characterized by site-dependent (varying) bilinear and biquadratic exchange interactions can be written as

$$
\mathcal{H}=-\sum_{i}\left[J_{e} f_{i}\left(\mathbf{S}_{i} . \mathbf{S}_{i+1}\right)+J_{b} g_{i}\left(\mathbf{S}_{i} . \mathbf{S}_{i+1}\right)^{2}\right]
$$

where $\mathbf{S}_{i}=\left(S_{i}^{x}, S_{i}^{y}, S_{i}^{z}\right)$ represents the classical three component spin vector and $J_{e}$ and $J_{b}$ respectively are the bilinear and biquadratic exchange parameters. $f_{i}$ and $g_{i}$ characterize the variation of the bilinear and biquadratic exchange interactions along the spin chain. The equation of motion corresponding to the spin Hamiltonian (2.1) can be constructed from [1]

$$
\frac{d \mathbf{S}_{i}}{d t}=\left\{\mathbf{S}_{i}, \mathcal{H}\right\}_{P B}
$$

The Poisson bracket in the right-hand side of Eq.(2.2) for any two arbitrary functions $\mathrm{F}$ and $\mathrm{H}$ of spins is defined as

$$
\{F, H\}_{P B}=\sum_{i=1}^{N} \sum_{\alpha, \beta, \gamma=1}^{3} \epsilon_{\alpha \beta \gamma} \frac{\partial F}{\partial S_{i}^{\alpha}} \frac{\partial H}{\partial S_{i}^{\beta}} S_{i}^{\gamma},
$$

where $\epsilon_{\alpha \beta \gamma}$ is the complete antisymmetric Levi-Civita tensor. The above spin Poisson bracket satisfies the same algebraic relations as the usual canonical Poisson bracket. On using our spin Hamiltonian (2.1) in Eq.(2.2), we obtain the following discrete equation of motion for the spins.

$$
\begin{aligned}
\frac{d \mathbf{S}_{n}}{d t}= & \mathbf{S}_{n} \wedge\left\{J_{e}\left(f_{n} \mathbf{S}_{n+1}+f_{n-1} \mathbf{S}_{n-1}\right)+J_{b}\left(g_{n}\left(\mathbf{S}_{n} \cdot \mathbf{S}_{n+1}\right) \mathbf{S}_{n+1}\right.\right. \\
& \left.\left.+g_{n-1}\left(\mathbf{S}_{n} \cdot \mathbf{S}_{n-1}\right) \mathbf{S}_{n-1}\right)\right\} .
\end{aligned}
$$

The structure of Eq.(2.4) demands that the length of the spin vector does not change with time and hence all the spins are assumed to have unit length $\left(\mathbf{S}_{n}^{2}=1\right)$.

Now, in order to understand the spin dynamics, we have to solve the discrete spin equation (2.4). However, in the low temperature and long wavelength limit one can go to the continuum limit by assuming that the lattice constant is very small compared to the length of the lattice. We assume that the spins $\mathbf{S}_{n}$ and the exchange coefficients $f_{n}$ and $g_{n}$ vary slowly over different distance scales " $a$ "(lattice distance) and " $b "$ respectively. Thus, we introduce the following series expansions for $\mathbf{S}_{n \pm 1}$ and $f_{n-1}, g_{n-1}$ by assuming $\mathbf{S}_{n}(t), f_{n}$ and $g_{n}$ respectively as $\mathbf{S}(x, t), f(x)$ and $g(x)$ where $x$ is a continuous variable. 


$$
\begin{aligned}
\mathbf{S}_{n \pm 1} & =\mathbf{S}(x, t) \pm a \frac{\partial \mathbf{S}}{\partial x}+\frac{a^{2}}{2 !} \frac{\partial^{2} \mathbf{S}}{\partial x^{2}} \pm \frac{a^{3}}{3 !} \frac{\partial^{3} \mathbf{S}}{\partial x^{3}}+\frac{a^{4}}{4 !} \frac{\partial^{4} \mathbf{S}}{\partial x^{4}}+\ldots \\
\left(\begin{array}{c}
f_{n-1} \\
g_{n-1}
\end{array}\right) & =\left(\begin{array}{l}
f(x) \\
g(x)
\end{array}\right)-b \frac{\partial}{\partial x}\left(\begin{array}{l}
f \\
g
\end{array}\right)+\frac{b^{2}}{2 !} \frac{\partial^{2}}{\partial x^{2}}\left(\begin{array}{l}
f \\
g
\end{array}\right)-\ldots
\end{aligned}
$$

It may be noted that in Eqs.(2.5), the spins have been expanded to $O\left(a^{4}\right)$ and the exchange coefficients only upto $O\left(b^{2}\right)$. In the normal cases " $a$ " and " $b$ " are expected to coincide with each other. But, nevertheless there may be situations where $b \neq a$. Using the above expansions in the discrete equation of motion (2.4), we obtain the following continuous equation of motion written to $O\left(a^{l} b^{m}\right), l+m=4$.

$$
\begin{aligned}
\mathbf{S}_{t}= & \mathbf{S} \wedge\left\{\left[A-\frac{b}{2} A_{x}+\frac{b^{2}}{2} A_{x x}+\frac{a^{2}}{2} J_{b} g\left(\mathbf{S} . \mathbf{S}_{x x}\right)\right] \mathbf{S}_{x x}+\left[\frac{b}{a} A_{x}-\frac{b^{2}}{2 a} A_{x x}\right.\right. \\
& \left.\left.+\frac{a^{2}}{3} J_{b} g\left(\mathbf{S} . \mathbf{S}_{x x x}\right)\right] \mathbf{S}_{x}+\frac{a^{2}}{12} A \mathbf{S}_{x x x x}+\frac{a b}{6} A_{x} \mathbf{S}_{x x x}\right\}, \quad \mathbf{S}^{2}=1,
\end{aligned}
$$

where $A(x)=J_{e} f(x)+J_{b} g(x)$. In Eq.(2.6), the suffices t and $\mathrm{x}$ represent partial derivatives with respect to $\mathrm{t}$ and $\mathrm{x}$ respectively. Eq.(2.6) describes the dynamics of spins in a one dimensional classical continuum isotropic inhomogeneous biquadratic Heisenberg spin chain which is in the form of the L-L[1] equation $\mathbf{S}_{t}=\mathbf{S} \wedge \mathbf{F}_{e f f}$, where $\mathbf{F}_{e f f}=\left[A-\frac{b}{2} A_{x}+\frac{b^{2}}{2} A_{x x}+\frac{a^{2}}{2} J_{b} g\left(\mathbf{S} . \mathbf{S}_{x x}\right)\right] \mathbf{S}_{x x}+$ $\left.\left[\frac{b}{a} A_{x}-\frac{b^{2}}{2 a} A_{x x}+\frac{a^{2}}{3} J_{b} g\left(\mathbf{S} . \mathbf{S}_{x x x}\right)\right] \mathbf{S}_{x}+\frac{a^{2}}{12} A \mathbf{S}_{x x x x}+\frac{a b}{6} A_{x} \mathbf{S}_{x x x}\right]$.

\section{A GENERALIZED NLS EQUATION AND SPIN DYNAMICS}

In order to understand the nonlinear spin dynamics of the inhomogeneous isotropic biquadratic Heisenberg spin chain more transparently, we map the spin chain at a given instant of time to the moving space curve in $E^{3}[2,6]$. A local coordinate system $\mathbf{e}_{i}(i=1,2,3)$ is formed on the space curve by identifying the unit spin vector $\mathbf{S}(x, t)$ with the unit tangent vector $\mathbf{e}_{1}(x, t)$ of the space curve and defining the unit principal and binormal vectors $\mathbf{e}_{2}(x, t)$ and $\mathbf{e}_{3}(x, t)$ respectively in the usual way. The change in orientation of the trihedral $\mathbf{e}_{i},(i=1,2,3)$ which defines the space curve uniquely within rigid motions is determined by the Serret-Frenet(S-F) equations[14]

$$
\left(\begin{array}{c}
\mathbf{e}_{1 x} \\
\mathbf{e}_{2 x} \\
\mathbf{e}_{3 x}
\end{array}\right)=\left(\begin{array}{ccc}
0 & \kappa & 0 \\
-\kappa & 0 & \tau \\
0 & -\tau & 0
\end{array}\right)\left(\begin{array}{l}
\mathbf{e}_{1} \\
\mathbf{e}_{2} \\
\mathbf{e}_{3}
\end{array}\right),
$$

where $\kappa \equiv\left(\mathbf{e}_{1 x} \cdot \mathbf{e}_{1 x}\right)^{\frac{1}{2}}$ and $\tau \equiv \frac{1}{\kappa^{2}} \mathbf{e}_{1} \cdot\left(\mathbf{e}_{1 x} \wedge \mathbf{e}_{1 x x}\right)$ are the curvature and torsion of the space curve. In view of the above identification and using the S-F equations (3.1), the equation of motion (2.6) 
can be rewritten as

$$
\begin{aligned}
\mathbf{e}_{1 t}= & \left\{-\tilde{h} \kappa \tau-\frac{a^{2}}{12} A\left(3 \kappa_{x x} \tau+3 \kappa_{x} \tau_{x}-\kappa^{3} \tau+\kappa \tau_{x x}-\kappa \tau^{3}\right)-\frac{a b}{6} A_{x}\left(2 \kappa_{x} \tau+\kappa \tau_{x}\right)\right. \\
& \left.+\frac{a^{2}}{2} J_{b} g \kappa^{3} \tau\right\} \mathbf{e}_{2}+\left\{\tilde{h} \kappa_{x}+\frac{a^{2}}{12} A\left(\kappa_{x x x}-6 \kappa^{2} \kappa_{x}-3 \kappa_{x} \tau^{2}-3 \kappa \tau \tau_{x}\right)\right. \\
& \left.+\frac{a b}{6} A_{x}\left(\kappa_{x x}-\kappa^{3}-\kappa \tau^{2}\right)+\tilde{h}_{x} \kappa-3 a^{2} J_{b} g \kappa^{2} \kappa_{x}\right\} \mathbf{e}_{3} .
\end{aligned}
$$

where $\tilde{h}=\left(A-\frac{b}{2} A_{x}+\frac{b^{2}}{2} A_{x x}\right)$. The time evolution of the normals $\mathbf{e}_{i}, i=1,2,3$ can be evaluated by using the S-F equations and Eq.(3.2). Thus after some lengthy algebra $\mathbf{e}_{i t}, i=1,2,3$ can be rewritten as

$$
\left(\begin{array}{l}
\mathbf{e}_{1 t} \\
\mathbf{e}_{2 t} \\
\mathbf{e}_{3 t}
\end{array}\right)=\left(\begin{array}{ccc}
0 & \omega_{3} & -\omega_{2} \\
-\omega_{3} & 0 & \omega_{1} \\
\omega_{2} & -\omega_{1} & 0
\end{array}\right)\left(\begin{array}{l}
\mathbf{e}_{1} \\
\mathbf{e}_{2} \\
\mathbf{e}_{3}
\end{array}\right)
$$

where

$$
\begin{aligned}
\omega_{1}= & \frac{1}{\kappa}\left\{\tilde{h}_{x} \kappa_{x}+\tilde{h}\left(\kappa_{x x}-\kappa \tau^{2}\right)+\left(\tilde{h}_{x} \kappa\right)_{x}+\frac{a^{2}}{2} J_{b} g\left(\kappa^{3} \tau^{2}-6 \kappa \kappa_{x}^{2}-3 \kappa^{2} \kappa_{x x}\right)+\frac{a^{2}}{12} A\left(\kappa_{x x x x}-12 \kappa \kappa_{x}^{2}\right.\right. \\
& \left.-6 \kappa^{2} \kappa_{x x}-6 \kappa_{x x} \tau^{2}-12 \kappa_{x} \tau_{x} \tau-3 \kappa \tau_{x}^{2}-4 \kappa \tau \tau_{x x}+\kappa^{3} \tau^{2}+\kappa \tau^{4}\right)+\frac{a b}{4} A_{x}\left(\kappa_{x x x}\right. \\
& \left.\left.-4 \kappa^{2} \kappa_{x}-3 \kappa_{x} \tau^{2}-3 \kappa \tau \tau_{x}\right)+\frac{a b}{12} A_{x x}\left(\kappa_{x x}-\kappa^{3}-\kappa \tau^{2}\right)-a b J_{b} g_{x} \kappa^{2} \kappa_{x}\right\} \\
\omega_{2}= & (\tilde{h} \kappa)_{x}+\frac{a^{2}}{12} A\left(\kappa_{x x x}-6 \kappa^{2} \kappa_{x}-3 \kappa_{x} \tau^{2}-3 \kappa \tau \tau_{x}\right)+\frac{a b}{6} A_{x}\left(\kappa_{x x}-\kappa^{3}-\kappa \tau^{2}\right) \\
& -\frac{3}{2} a^{2} J_{b} g \kappa^{2} \kappa_{x} \\
\omega_{3}= & -\tilde{h} \kappa \tau-\frac{a^{2}}{12} A\left(3 \kappa_{x x} \tau+3 \kappa_{x} \tau_{x}-\kappa^{3} \tau+\kappa \tau_{x x}-\kappa \tau^{3}\right)-\frac{a b}{6} A_{x}\left(2 \kappa_{x} \tau+\kappa \tau_{x}\right) \\
& +\frac{a^{2}}{2} J_{b} g \kappa^{3} \tau
\end{aligned}
$$

Now, the compatibility of equations (3.1) and (3.3) namely $\left(\mathbf{e}_{i x}\right)_{t}=\left(\mathbf{e}_{i t}\right)_{x}$ lead to the following evolution equations for the curvature and torsion of the space curve.

$$
\begin{aligned}
\kappa_{t}= & -\tilde{h}\left(2 \kappa_{x} \tau+\kappa \tau_{x}\right)-2 \tilde{h}_{x} \kappa \tau-\frac{a^{2}}{12} A\left(4 \kappa_{x x x} \tau+6 \kappa_{x x} \tau_{x}-4 \kappa_{x} \tau^{3}-6 \kappa \tau^{2} \tau_{x}+4 \kappa_{x} \tau_{x x}\right. \\
& \left.+\kappa \tau_{x x x}-9 \kappa^{2} \kappa_{x} \tau-\kappa^{3} \tau_{x}\right)-\frac{a b}{4} A_{x}\left(3 \kappa_{x x} \tau+3 \kappa_{x} \tau_{x}-\kappa^{3} \tau+\kappa \tau_{x x}-\kappa \tau^{3}\right) \\
& -\frac{a^{2}}{2} J_{b} g\left(6 \kappa^{2} \kappa_{x} \tau+\kappa^{3} \tau_{x}\right)
\end{aligned}
$$




$$
\begin{aligned}
\tau_{t}= & \left\{\frac{\kappa_{x x}}{\kappa}-\tau^{2}+\frac{\kappa^{2}}{2}+\frac{a^{2}}{12} A\left(\frac{\kappa_{x x x x}}{\kappa}-5 \kappa_{x x} \kappa-\frac{25}{2} \kappa_{x}^{2}-4 \tau \tau_{x x}-\frac{6 \tau^{2} \kappa_{x x}}{\kappa}-3 \tau_{x}^{2}-\frac{3 \tau_{x}^{4}}{2}\right.\right. \\
& \left.\left.-\frac{12 \kappa_{x} \tau \tau_{x}}{\kappa}+\tau^{4}-\frac{\kappa^{2} \tau^{2}}{2}\right)+\frac{a^{2}}{2} J_{b} g\left(\kappa^{2} \tau^{2}-6 \kappa_{x}^{2}-3 \kappa \kappa_{x x}-\frac{3 \kappa^{4}}{4}\right)\right\}_{x}+A_{x}\left[\left(\kappa^{2}-\frac{\kappa_{x}^{2}}{\kappa^{2}}\right.\right. \\
& \left.+\frac{3 \kappa_{x x}}{\kappa}-\tau^{2}\right)+\frac{b}{2}\left(-\kappa \kappa_{x}+\frac{\kappa_{x x} \kappa_{x}}{\kappa^{2}}-\frac{\kappa_{x x x}}{\kappa}+2 \tau \tau_{x}\right)+\frac{a b}{12}\left(-4 \kappa \kappa_{x x}+\frac{\kappa^{4}}{16}-3 \kappa^{2} \tau^{2}\right. \\
& -\frac{3 \kappa_{x} \kappa_{x x x}}{\kappa^{2}}-\frac{3 \kappa_{x} \kappa_{x x x x}}{\kappa^{2}}-\frac{3 \kappa_{x}^{2} \tau^{2}}{\kappa^{2}}+\frac{9 \kappa_{x} \tau \tau_{x x}}{\kappa}-\frac{3 \kappa_{x} \tau \tau_{x}}{\kappa}+\frac{\kappa_{x x x x}}{\kappa}+\frac{\kappa_{x}^{3}}{\kappa}+12 \kappa_{x} \kappa_{x x} \\
& \left.\left.-\frac{3 \kappa_{x} \kappa_{x x} \tau^{2}}{\kappa^{2}}-\frac{12 \kappa_{x}^{2} \tau \tau_{x}}{\kappa^{2}}+\frac{9 \kappa_{x} \tau_{x}^{2}}{\kappa}-\frac{6 \kappa_{x x} \tau^{2}}{\kappa}-3 \tau_{x}^{2}-4 \tau \tau_{x x}+\tau^{4}\right)\right]+A_{x x}\left[\frac{2 \kappa_{x}}{\kappa}\right. \\
& +\frac{b}{2}\left(\kappa^{2}-\frac{\kappa_{x}^{2}}{\kappa^{2}}+\frac{3 \kappa_{x x}}{\kappa}-\tau^{2}\right)+\frac{b^{2}}{12}\left(-10 \kappa \kappa_{x}-\frac{8 \kappa_{x x} \kappa_{x}}{\kappa^{2}}+\frac{11 \kappa_{x x x}}{\kappa}-25 \tau \tau_{x}\right. \\
& \left.\left.-9 \frac{\tau^{2} \kappa_{x}}{\kappa}\right)\right] .
\end{aligned}
$$

The curvature and torsion of the space curve are related to the energy and current densities of the spin system and thus the spin dynamics is equivalently represented in terms of the evolution of the curvature and torsion of the space curve. In order to identify the set of coupled equations (3.5) and (3.6) with more standard nonlinear partial differential equations, we make the complex transformation [3]

$$
q=\frac{\kappa}{2} \exp i \int_{-\infty}^{x} \tau\left(x^{\prime}, t\right) d x^{\prime},
$$

and obtain the following inhomogeneous generalized NLS equation.

$$
\begin{aligned}
& i q_{t}+(h q)_{x x}+2 h|q|^{2} q+2 q \int_{-\infty}^{x} h_{x^{\prime}}|q|^{2} d x^{\prime}+\frac{a^{2} A}{12}\left[q_{x x x x}+K_{1}|q|^{2} q_{x x}\right. \\
& \left.+K_{2} q^{2} q_{x x}^{*}+K_{3} q\left|q_{x}\right|^{2}+K_{4} q^{*} q_{x}^{2}+3 K_{2} q|q|^{4}\right]+\frac{a b}{12} A_{x}\left[q_{x x x}+6|q|^{2} q_{x}\right]=0 .
\end{aligned}
$$

where $h(x)=\tilde{h}+\frac{b^{2}}{6} A_{x x}, K_{1}=-12\left(1+\frac{4 J_{b} g}{A}\right), \quad K_{2}=-8\left(1+\frac{3 J_{b} g}{A}\right), \quad K_{3}=-36\left(1+\frac{8 J_{b} g}{3 A}\right), \quad K_{4}=$ $-14\left(1+\frac{24 J_{b} g}{7 A}\right)$.

At different orders of the expansion parameters and for different A, Eq.(3.8) reduces to different completely integrable equations of the NLS family. For example, at the lowest order, i.e. at $O\left(a^{0} b^{0}\right)$, when $\mathrm{A}$ is a constant, Eq.(3.8) reduces to the completely integrable cubic NLS equation

$$
i q_{t}+q_{x x}+2|q|^{2} q=0,
$$

and at the same order when $\mathrm{A}$ is a linear function of $\mathrm{x}$ it reduces to the following inhomogeneous cubic NLS equation

$$
i q_{t}+2 C q_{x}+(C x+D)\left[q_{x x}+2 q|q|^{2}\right]+2 C q \int_{-\infty}^{x}|q|^{2} d x^{\prime}=0 .
$$

At $O\left(a^{2}\right)$, when $a=b$ and $A=$ constant and for the choice $\frac{J_{b} g}{A}=\frac{-5}{12}$, Eq.(3.8) gives the following completely integrable fourth order NLS equation.

$$
\begin{aligned}
& i q_{t}+q_{x x}+2 q|q|^{2}+\frac{a^{2}}{12}\left[q_{x x x x}+8|q|^{2} q_{x x}\right. \\
& \left.+2 q^{2} q_{x x}^{*}+4\left|q_{x}\right|^{2} q+6 q^{*} q_{x}^{2}+6|q|^{4} q\right]=0 .
\end{aligned}
$$


The above set of three completely integrable NLS family of equations were obtained from isotropic bilinear[3], isotropic inhomogeneous[11,12] and isotropic biquadratic[5,6] Heisenberg spin chains respectively through Lakshmanan's space curve mapping procedure. The corresponding LL equation of motion for the completely integrable spin system can be written as follows.

(i) Isotropic bilinear Heisenberg spin chain[3].

$$
\mathbf{S}_{t}=\mathbf{S} \wedge \mathbf{S}_{x x}
$$

(ii) Isotropic inhomogeneous bilinear Heisenberg spin chain[11,12].

$$
\mathbf{S}_{t}=\mathbf{S} \wedge\left[(C x+D) \mathbf{S}_{x x}+C \mathbf{S}_{x}\right]
$$

(iii) Isotropic biquadratic Heisenberg spin chain $[5,6]$.

$$
\mathbf{S}_{t}=\mathbf{S} \wedge\left[\mathbf{S}_{x x}+\frac{a^{2}}{12}\left[\mathbf{S}_{x x x x}-\frac{5}{2}\left(\mathbf{S} . \mathbf{S}_{x x}\right) \mathbf{S}_{x x}-\frac{5}{3}\left(\mathbf{S} . \mathbf{S}_{x x x}\right) \mathbf{S}_{x}\right]\right.
$$

The integrability properties of the above set of completely integrable equations have been studied in detail and the spin excitations in the above spin models are governed by spin solitons.

An inspection of Eq.(3.8) reveals that in addition to the above, it contains at least two inhomogeneous higher order equations which may lead to completely integrable spin models with soliton spin excitations. For instance, in the next higher order assuming that $O(a b)<O\left(a^{2}\right)$ and redefining $a \rightarrow i a$ we have the following inhomogeneous Hirota-type equation.

$$
i q_{t}+(h q)_{x x}+2 h q|q|^{2}+2 q \int_{-\infty}^{x} h_{x^{\prime}}|q|^{2} d x^{\prime}+\frac{i a b}{12} A_{x}\left[q_{x x x}+6|q|^{2} q_{x}\right]=0 .
$$

Eq.(3.15) with $\mathrm{A}(\mathrm{x})$ in the form of a linear function of $\mathrm{x}$ has been studied in the context of a generalized $\mathrm{x}$-dependent Hirota equation by carrying out singularity structure analysis and the integrability properties investigated by constructing the Lax pair and soliton solutions[15]. Finally, at $O\left(a^{2}\right)$, assuming that $O(a b)>O\left(a^{2}\right)$, Eq.(3.7) gives the following inhomogeneous fourth order NLS-type equation.

$$
\begin{aligned}
& i q_{t}+(h q)_{x x}+2 h q|q|^{2}+2 q \int_{-\infty}^{x} h_{x^{\prime}}|q|^{2} d x^{\prime}+\frac{a^{2} A}{12}\left[q_{x x x x}+K_{1}|q|^{2} q_{x x}\right. \\
& \left.+K_{2} q^{2} q_{x x}^{*}+K_{3} q\left|q_{x}\right|^{2}+K_{4} q^{*} q_{x}^{2}+3 K_{2} q|q|^{4}\right]=0 .
\end{aligned}
$$

Now we seek whether Eq.(3.16) is also expected to be integrable only when A is a linear function of $\mathrm{x}$ or otherwise. If so next we will analyse to see what would happen to the spin excitations when $\mathrm{A}(\mathrm{x})$ and hence the inhomogeneity arises in the form of a nonlinear function. This forms the major task to be carried out in the rest of the paper. We investigate these aspects by carrying out Painlevé singularity structure analysis followed by multiple scale perturbation analysis.

\section{SINGULARITY STRUCTURE ANALYSIS AND SPIN SOLITON}

In order to pick up completely integrable models underlying Eq.(3.16), so that the elementary spin excitations can be expressed in terms of solitons, we carry out Painlevé singularity structure 
analysis[16,17]. The Painlevé analysis is a useful tool to verify whether the given nonlinear partial differential equation is free from movable critical manifolds so that the generalized Painlevé property holds. This procedure ensures the single valuedness of the general solution of the given nonlinear partial differential equation around any noncharacteristic movable singular manifold when expressed locally as a Laurent series. The singularity structure analysis which proceeds along three major steps namely (i) finding the leading order behaviour (ii) identifying resonances and (iii) verifying the existence of sufficient number of arbitrary functions is now an established procedure and hence we do not present the complete details of the analysis here.

In the following, we rewrite Eq.(3.16) and its complex conjugate equation by denoting $q$ and $q^{*}$ by $\mathrm{E}$ and $\mathrm{G}$ respectively and defining a new real function $\mathrm{R}$.

$$
\begin{aligned}
& i E_{t}+E_{x x}+2 h_{x} E_{x}+h_{x x} E+2 R E+\frac{a^{2}}{12} A\left[E_{x x x x}+K_{1} E G E_{x x}+K_{2} E^{2} G_{x x}\right. \\
& \left.+K_{3} E E_{x} G_{x}+K_{4} G E_{x}^{2}+3 K_{2} E^{3} G^{2}\right]=0, \\
& -i G_{t}+G_{x x}+2 h_{x} G_{x}+h_{x x} G+2 R G+\frac{a^{2}}{12} A\left[G_{x x x x}+K_{1} E G G_{x x}+K_{2} G^{2} E_{x x}\right. \\
& \left.+K_{3} G E_{x} G_{x}+K_{4} E G_{x}^{2}+3 K_{2} E^{2} G^{3}\right]=0, \\
& R_{x}-2 h_{x} E G-h(E G)_{x}=0 .
\end{aligned}
$$

We perform Painlevé singularity structure analysis on Eqs.(4.1) by expanding the functions E, G and $\mathrm{R}$ locally in the form of the Laurent series

$$
\begin{aligned}
& E=E_{0}(x, t) \phi^{p}(x, t)+\sum_{j=1} E_{j}(x, t) \phi^{p+j} \\
& G=G_{0}(x, t) \phi^{s}(x, t)+\sum_{j=1} G_{j}(x, t) \phi^{s+j} \\
& R=R_{0}(x, t) \phi^{w}(x, t)+\sum_{j=1} R_{j}(x, t) \phi^{w+j} .
\end{aligned}
$$

Now using the leading order terms of the solutions (4.2), i.e. $E \sim E_{0}(x, t) \phi^{p}(x, t), G \sim$ $G_{0}(x, t) \phi^{s}(x, t), R \sim R_{0}(x, t) \phi^{w}(x, t)$ in Eqs.(4.1) and on balancing the dominant terms, we obtain the following two branches of leading order behaviour.

$\operatorname{Branch}(\mathrm{i})$

$$
\begin{aligned}
p & =s=-1, w=-2, \\
E_{0} G_{0} & =\frac{-\phi_{x}^{2}}{4\left(1+\frac{3 J_{b} g}{A}\right)}, R_{0}=\frac{-h \phi_{x}^{2}}{4\left(1+\frac{3 J_{b} g}{A}\right)} .
\end{aligned}
$$

Branch (ii)

$$
\begin{aligned}
p & =s=-1, w=-2, \\
E_{0} G_{0} & =-4 \phi_{x}^{2}, R_{0}=-4 h \phi_{x}^{2} .
\end{aligned}
$$

Now to find the resonances i.e. the powers at which free coefficients enter into the generalized 
expansion, we expand

$$
\begin{aligned}
& E=E_{0} \phi^{p}+\ldots+\alpha_{1} \phi^{p+r} \\
& G=G_{0} \phi^{s}+\ldots+\alpha_{2} \phi^{s+r} \\
& R=R_{0} \phi^{w}+\ldots+\alpha_{3} \phi^{w+r}
\end{aligned}
$$

and substitute in Eqs.(4.1) containing the leading order terms alone. The result yields

$$
\left(\begin{array}{clc}
M_{1} & E_{0}^{2} M_{2} & 0 \\
G_{0}^{2} M_{2} & M_{1} & 0 \\
h G_{0}(2-r) & h E_{0}(2-r) & r-2
\end{array}\right)\left(\begin{array}{c}
\alpha_{1} \\
\alpha_{2} \\
\alpha_{3}
\end{array}\right)=0
$$

where $M_{1}=(r-1)(r-2)(r-3)(r-4)+\left[(r-1)\left(K_{1}(r-2)-K_{3}-2 K_{4}\right)+2 K_{1}+4 K_{2}+K_{3}\right] E_{0} G_{0}+$ $9 K_{2} E_{0}^{2} G_{0}^{2}$ and $M_{2}=\left[K_{2}(r-1)(r-2)-K_{3}(r-1)+K_{4}+2 K_{1}\right]+6 K_{2} E_{0} G_{0}$. On substituting $E_{0} G_{0}$, in the resonance matrix (4.7), we obtain all real resonances only for the following set of parametric choices $(a) \frac{J_{b} g}{A}=\frac{-17}{48}$ and $(b) \frac{J_{b} g}{A}=\frac{-5}{12}$. In the case (a) the two branches merge into a single branch with the leading order results $E_{0} G_{0}=-4 \phi_{x}^{2}, R_{0}=-4 h \phi_{x}^{2}$ and the resonances, $r=-1,-1,0,0,2,3,4,7,8$. Analysing further, we find that the system admits a logarithmic singularity manifold at $r=0$, thereby destroying the Painlevé nature of solution and therefore we stop treating case(a) further. For the case (b) we obtain the following two branches.

Branch(i)

$$
E_{0} G_{0}=-\phi_{x}^{2}, \quad R_{0}=-h \phi_{x}^{2}, r=-1,0,1,2,2,3,4,5,6
$$

$\operatorname{Branch}(\mathrm{ii})$

$$
E_{0} G_{0}=-4 \phi_{x}^{2}, R_{0}=-4 h \phi_{x}^{2}, r=-3,-2,-1,0,2,5,6,7,8
$$

In both the branches the resonances -1 and 0 correspond to the arbitrariness of the manifold and of $E_{0}$ or $G_{0}$ respectively.

To check whether sufficient number of arbitrary functions exist without introducing movable critical singularity manifolds, we substitute the Laurent expansion (4.2) in the full equation (4.1) and collect coefficients of different powers of $\phi$ and obtain $R_{1}=-2 h_{x}+h \phi_{x x}, R_{2}=$ arbitrary and the following compatibility conditions corresponding to different resonances. $\left(\phi^{-4}, \phi^{-4} \phi^{-2}\right): r=1$ :

$$
E_{0} G_{1}+G_{0} E_{1}=\phi_{x x} .
$$

$\left(\phi^{-3}, \phi^{-3} \phi^{-1}\right): \mathrm{r}=2,2$ :

$$
\begin{aligned}
E_{0} G_{2}-G_{0} E_{2}= & \frac{1}{6 \phi_{x}^{2}}\left[-3\left(E_{0} G_{0 x x}-G_{0} E_{0 x x}\right)-2\left(\phi_{x} \phi_{x x}\right)_{x}-4 \phi_{x x}^{2}+4\left(2 G_{0} E_{1 x}\right.\right. \\
& \left.\left.-E_{0} G_{1 x}\right) \phi_{x}+4\left(G_{0 x} \phi_{x}-G_{0} \phi_{x x}\right) E_{1}+8 E_{0 x} \phi_{x} \phi_{x x}\right] \\
h_{x x}= & 0 .
\end{aligned}
$$


From the above results, we observe that $E_{1}$ or $G_{1}$ and $E_{2}$ or $G_{2}$ and $R_{2}$ are arbitrary in addition to the condition that $h_{x x}=0$ implying that $\mathrm{h}$ is a linear function of $\mathrm{x}$, say $h=C x+D$ where $\mathrm{C}$ and $\mathrm{D}$ are constants. Proceeding further, we find that sufficient number of arbitrary functions enter without introduction of movable critical manifolds at $r=3,4,5$ and 6 . However, as the compatibility conditions corresponding to these resonances are unwieldy in nature we are not presenting them here. For the branch (ii), we verify that arbitrary functions enter at $r=5,6,7$ and 8. Thus Eq.(4.1) satisfies Painlevé property and is a candidate to be integrable when $\frac{J_{b} g}{A}=\frac{-5}{12}$ and $h=C x+D$. The integrable form of Eq.(3.16) can therefore be written as

$$
\begin{aligned}
& i q_{t}+2 C q_{x}+(C x+D)\left[q_{x x}+2|q|^{2} q\right]+2 C q \int_{-\infty}^{x}|q|^{2} d x^{\prime}+\frac{a^{2}}{12}\left[q_{x x x x}\right. \\
& \left.+8|q|^{2} q_{x x}+2 q^{2} q_{x x}^{*}+4\left|q_{x}\right|^{2} q+6 q^{*} q_{x}^{2}+6 q|q|^{4}\right]=0 .
\end{aligned}
$$

The corresponding spin equation is written as

$$
\mathbf{S}_{t}=\mathbf{S} \wedge\left[\left[(C x+D) \mathbf{S}_{x x}+C \mathbf{S}_{x}\right]+\frac{a^{2}}{12}\left[\mathbf{S}_{x x x x}-\frac{5}{2}\left(\mathbf{S} . \mathbf{S}_{x x}\right) \mathbf{S}_{x x}-\frac{5}{3}\left(\mathbf{S} . \mathbf{S}_{x x x}\right) \mathbf{S}_{x}\right]\right] .
$$

The integrability properties of Eqs.( 4.12) and (4.13) in the absence of inhomogeneity have been discussed in detail in refs.[5,6]. When linear inhomogeneity is present in the system the Lax pair and soliton solutions can be written as follows[5,6,15]. The Lax pair U, V associated with the AKNS-type linear eigenvalue problem $\Psi_{x}=U \Psi, \Psi_{t}=V \Psi$, where $\Psi=\left(\Psi_{1}, \Psi_{2}\right)^{T}$ corresponding to Eq.(4.12) can be written as[18]

$$
\begin{aligned}
U= & \left(\begin{array}{cc}
-i \hat{\lambda} & q \\
-q^{*} & i \hat{\lambda}
\end{array}\right) \\
V= & {\left[i|q|^{2}+i \hat{\gamma}\left[q^{*} q_{x x}+q q_{x x}^{*}-\left|q_{x}\right|^{2}+3|q|^{4}\right]+2 \hat{\lambda} \hat{\gamma}\left(q q_{x}^{*}-q_{x} q *\right)-2 i \hat{\lambda}^{2}[1+C x+D\right.} \\
& \left.\left.+2 \hat{\gamma}|q|^{2}\right]+8 i \hat{\gamma} \hat{\lambda}^{4}\right] \sigma_{z}+i \sigma_{z} \mathcal{M}_{x}+i \hat{\gamma}\left[6 \mathcal{M}^{2} \mathcal{M}_{x} \sigma_{z}+\sigma_{z} \mathcal{M}_{x x x}\right]-4 i \hat{\gamma} \lambda^{2} \sigma_{z} \mathcal{M}_{x} \\
& +2(C x+D) \mathcal{M}+2 \hat{\lambda}\left[\mathcal{M}+\hat{\gamma}\left(\mathcal{M}_{x x}-2 \mathcal{M}^{3}\right)\right]-8 \hat{\gamma} \hat{\lambda}^{3} \mathcal{M}
\end{aligned}
$$

where $\hat{\lambda}$ is the spectral parameter and $\hat{\gamma}=\frac{a^{2} A}{12}, \sigma_{z}=\left(\begin{array}{cc}1 & 0 \\ 0 & -1\end{array}\right), \mathcal{M}=\left(\begin{array}{cc}0 & q \\ -q^{*} & 0\end{array}\right)$. Knowing the Lax pair the multisoliton solutions can be constructed using the inverse scattering transform (IST) method[19] or using the Bäcklund transformation technique[20,21]. For example, the one soliton solution of Eq.(4.12) is found to be

$$
q(1)=-2 \rho \operatorname{sech} \zeta \exp (-2 i \varepsilon)
$$

where

$$
\begin{aligned}
& \zeta=2\left[\rho\left(\hat{\delta}_{1}+x\right)-32 \hat{\gamma} \mu^{2}+4\left(8 \rho^{2}+1\right) \mu \rho t\right]+8 D \int\left(\mu^{2}-\rho^{2}\right) d t+\hat{\delta}_{1}, \\
& \varepsilon=\mu\left(\hat{\delta}_{2}+x\right)+2\left[\left(\mu^{2}-\rho^{2}\right) t-4 \hat{\gamma}\left(\mu^{4}+\rho^{4}\right) t+24 \mu^{2} \rho^{2} t\right]+4 D \int \mu \rho d t+\hat{\delta}_{2},
\end{aligned}
$$


where $\rho$ and $\mu$ correspond to the scattering parameter and $\hat{\delta}_{1}$ and $\hat{\delta}_{2}$ are phase constants. The corresponding spin components can be written as $[6,22,23]$

$$
\begin{aligned}
& S^{x}=\frac{-2 \rho}{\left(\mu^{2}+\rho^{2}\right)}\left[\mu \cos \left(\frac{\mu}{\rho}\right) \zeta-\rho \sin \left(\frac{\mu}{\rho}\right) \zeta \tanh \zeta\right] \operatorname{sech} \zeta, \\
& S^{y}=\frac{-2 \rho}{\left(\mu^{2}+\rho^{2}\right)}\left[\mu \sin \left(\frac{\mu}{\rho}\right) \zeta+\rho \cos \left(\frac{\mu}{\rho}\right) \zeta \tanh \zeta\right] \operatorname{sech} \zeta, \\
& S^{z}=1-\left[\frac{2 \rho^{2}}{\left(\mu^{2}+\rho^{2}\right)}\right] \operatorname{sech}^{2} \zeta .
\end{aligned}
$$

\section{EFFECT OF NONLINEAR INHOMOGENEITY UNDER PERTURBATION}

The results of the singularity structure analysis in the previous section showed that the inhomogeneous biquadratic spin system becomes completely integrable and the elementary spin excitations expressed in terms of solitons only when the exchange inhomogeneity appears in the form of a linear function. Now the question arises as to what will be the effect of a nonlinear inhomogeneity on the spin soliton. We try to find an answer for this question by carrying out a multiple scale perturbation analysis by considering the inhomogeneous cubic NLS equation at the lower order from the generalized NLS equation (3.8).

$$
i q_{t}+(h q)_{x x}+2 h|q|^{2} q+2 q \int_{-\infty}^{x} h_{x^{\prime}}|q|^{2} d x^{\prime}=0 .
$$

We have considered the equation at the lower order for the perturbation analysis because the inhomogeneity does not enter into the terms at $O\left(a^{2}\right)$ and hence it is sufficient to analyse Eq.(5.1) to understand the effect of inhomogeneity on the spin soliton. The effect of higher order terms i.e. terms at $O\left(a^{2}\right)$ (discreteness effect)(without inhomogeneity) on the NLS soliton as perturbation is studied in refs.[5,6]. When $\mathrm{h}=h_{0}=$ constant, Eq.(5.1) reduces to the completely integrable cubic NLS equation (3.9) and when $\mathrm{h}$ is a linear function of $\mathrm{x}$ say $h(x)=C x+D$, where $\mathrm{C}$ and $\mathrm{D}$ are constants, it reduces to the completely integrable inhomogeneous NLS equation. We now substitute

$$
h(x)=h_{0}+\lambda h_{1}(x)
$$

where $\lambda$ is a small parameter and $h_{1}$ is a nonlinear function of $\mathrm{x}$, in Eq.(5.1) and after suitable rescaling of $t$ and redefinition of $\lambda$ we get,

$$
i q_{t}+q_{x x}+2|q|^{2} q+\lambda\left[\left(h_{1} q\right)_{x x}+2 h_{1}|q|^{2} q+2 q \int_{-\infty}^{x} h_{1 x^{\prime}}|q|^{2} d x^{\prime}\right]=0 .
$$

\section{A. Perturbation Method}

We now carry out the multiple scale perturbation analysis laid down by Kodama and Ablowitz[24] by treating terms proportional to $\lambda$ in Eq.(5.3) as perturbation. Considering $q_{0}$ 
as the exact solution corresponding to the unperturbed part $(\lambda=0)$, we introduce certain fast variables $\Theta_{i}(i=1,2,3, \ldots, M)$ and a slow variable $T=\lambda t$ and parameters $P_{i}(i=1,2,3, \ldots, M)$ which depend on the slow variables[24,25]. The unperturbed solution $q_{0}$ in terms of these new variables then take the form

$$
q_{0}=\hat{q}_{0}\left(\Theta_{1}, \Theta_{2}, \ldots, \Theta_{M} ; T ; P_{1}, P_{2}, \ldots, P_{M}\right)
$$

However, for one soliton solution, it is enough to introduce only one fast variable. Now we expand $\mathrm{q}$ in terms of a power series in $\lambda$.

$$
q=q_{0}+\lambda q_{1}+\ldots
$$

Using Eq.(5.5) in the perturbed equation (5.3) and collecting the coefficients of different powers of $\lambda$, we obtain a system of linear differential equations with variable coefficients. By solving these equations recursively we obtain the perturbed solution upon taking care of the secularity problem appropriately and using the boundary conditions. In what follows we make use of this multiple scaling analysis to Eq.(5.3).

When $\lambda=0$, Eq.(5.3) reduces to the completely integrable cubic NLS equation (3.9) which possesses N-soliton solutions. The envelope one soliton solution of Eq.(3.9) can be written as

$$
q=\eta \operatorname{sech} \eta\left(\theta-\theta_{0}\right) \exp \left[i \xi\left(\theta-\theta_{0}\right)+i\left(\sigma-\sigma_{0}\right)\right]
$$

where

$$
\theta_{t}=-2 \xi, \quad \theta_{x}=1, \quad \sigma_{t}=\eta^{2}+\xi^{2}, \quad \sigma_{x}=0 .
$$

We write $\eta, \xi, \theta, \theta_{0}$ and $\sigma_{0}$ as functions of the new time scale $T=\lambda t$ now. Hence, the soliton solution of Eq.(5.3) can be written as

$$
q=\hat{q}(\theta, T ; \lambda) \exp \left[i \xi\left(\theta-\theta_{0}\right)+i\left(\sigma-\sigma_{0}\right)\right]
$$

Under the assumption of quasi-stationarity, Eq.(5.3) can be written as

$$
-\eta^{2} \hat{q}+\hat{q}_{\theta \theta}+2 \hat{q}^{2} \hat{q}^{*}=\lambda F(\hat{q})
$$

where

$$
\begin{aligned}
F(\hat{q})= & i\left[-\hat{q}_{T}+2 h_{1 \theta} \xi \hat{q}+2 h_{1} \xi \hat{q}_{\theta}\right]-\left[\xi_{T}\left(\theta-\theta_{0}\right)-\xi \theta_{0 T}\right]+\left(h_{1} \hat{q}\right)_{\theta \theta} \\
& -h_{1} \xi^{2} \hat{q}+2 h_{1}|\hat{q}|^{2} \hat{q}+2 \hat{q} \int_{-\infty}^{\theta} h_{1 \theta^{\prime}}|\hat{q}|^{2} d \theta^{\prime} .
\end{aligned}
$$

Expanding $\hat{q}$ in terms of $\lambda$ as

$$
\hat{q}(\theta, T ; \lambda)=\hat{q}_{0}(\theta, T)+\lambda \hat{q}_{1}(\theta, T)+\ldots
$$

where $\hat{q}_{0}=\eta \operatorname{sech} \eta\left(\theta-\theta_{0}\right)$. Substituting Eq.(5.10) in Eq.(5.8) at $O(\lambda)$ we get

$$
-\eta^{2} \hat{q}_{1}+\hat{q}_{1 \theta \theta}+2 \hat{q}_{0}^{2} \hat{q}_{1}^{*}+4 \hat{q}_{0}^{2} \hat{q}_{1}=F_{1}\left(\hat{q}_{0}\right)
$$


The right-hand side of Eq.(5.11a) is of the form

$$
\begin{aligned}
F_{1}\left(\hat{q}_{0}\right)= & i\left[-\hat{q}_{0 T}+2 h_{1 \theta} \xi \hat{q}_{0}+2 h_{1} \xi \hat{q}_{0 \theta}\right]-\left[\xi_{T}\left(\theta-\theta_{0}\right)-\xi \theta_{0 T}\right]+\left(h_{1} \hat{q}_{0}\right)_{\theta \theta} \\
& -h_{1} \xi^{2} \hat{q}_{0}+2 h_{1}\left|\hat{q}_{0}\right|^{2} \hat{q}_{0}+2 \hat{q}_{0} \int_{-\infty}^{\theta} h_{1 \theta^{\prime}}\left|\hat{q}_{0}\right|^{2} d \theta^{\prime}
\end{aligned}
$$

Substituting $\hat{q}_{1}=\hat{\phi}_{1}+i \hat{\psi}_{1}\left(\hat{\phi}_{1}\right.$ and $\hat{\psi}_{1}$ are real) in Eq. (5.11), we obtain

$$
\begin{aligned}
& \mathcal{L}_{1} \phi_{1} \equiv-\eta^{2} \phi_{1}+\phi_{1 \theta \theta}+6 \hat{q}_{0}^{2} \phi_{1}=\Re F_{1}\left(\hat{q}_{0}\right), \\
& \mathcal{L}_{2} \psi_{1} \equiv-\eta^{2} \psi_{1}+\psi_{1 \theta \theta}+2 \hat{q}_{0}^{2} \psi_{1}=\Im F_{1}\left(\hat{q}_{0}\right),
\end{aligned}
$$

where $\Re F_{1}\left(\hat{q}_{0}\right)$ and $\Im F_{1}\left(\hat{q}_{0}\right)$ are the real and imaginary parts of $F_{1}\left(\hat{q}_{0}\right)$ given by

$$
\begin{aligned}
\Re F_{1}\left(\hat{q}_{0}\right)= & -\left[\xi_{T}\left(\theta-\theta_{0}\right)-\xi \theta_{0 T}\right]+\left(h_{1} \hat{q}_{0}\right)_{\theta \theta} \\
& -h_{1} \xi^{2} \hat{q}_{0}+2 h_{1}\left|\hat{q}_{0}\right|^{2} \hat{q}_{0}+2 \hat{q}_{0} \int_{-\infty}^{\theta} h_{1 \theta^{\prime}}\left|\hat{q}_{0}\right|^{2} d \theta^{\prime}, \\
\Im F_{1}\left(\hat{q}_{0}\right)= & {\left[-\hat{q}_{0 T}+2 h_{1 \theta} \xi \hat{q}_{0}+2 h_{1} \xi \hat{q}_{0 \theta}\right], }
\end{aligned}
$$

and $\mathcal{L}_{1}$ and $\mathcal{L}_{2}$ are self-adjoint operators. On solving Eqs.(5.12) for specific form of inhomogeneity $h_{1}$, we obtain the perturbed soliton solution.

\section{B. Evolution of Amplitude and Velocity of the Soliton}

It may be noted that $\hat{q}_{0 \theta}$ and $\hat{q}_{0}$ are solutions of the homogeneous part of Eqs.(5.12a) and (5.12b) respectively and hence the secularity conditions yield

$$
\int_{-\infty}^{\infty} \hat{q}_{0 \theta} \Re F_{1} d \theta=0,
$$

and

$$
\int_{-\infty}^{\infty} \hat{q}_{0} \Im F_{1} d \theta=0 .
$$

Now, in order to carry out the integration in Eqs.(5.13) and (5.14), we have to supply the explicit form of the inhomogeneity $h_{1}$ in Eqs.(5.12c) and (5.12d). As the results of the Painlevé singularity structure analysis show that the inhomogeneous biquadratic spin chain model becomes integrable when the inhomogeneity appears in the form of a linear function of $\mathrm{x}$, we are interested in inohomogeneities other than the linear function and here we consider a simple nonlinear inhomogeneity in the form $h_{1}(x)=B x^{2}+C x+D$, where B, C and D are constants. Using the above form of $h_{1}$ in Eqs. (5.12c) and (5.12d) and making use of them and $\hat{q}_{0 \theta}$ and $\hat{q}_{0}$ in Eqs. (5.13) and (5.14) and integrating (for details, see Appendix A), we obtain

$$
\eta_{T}=2 K \xi(\eta-2)
$$

and

$$
\xi_{T}=2 K\left(\eta^{2}-\xi^{2}\right)
$$


where $K=(C+N)$ and $N=B \eta\left[\left(\theta-\theta_{0}\right) \tanh \eta\left(\theta-\theta_{0}\right)\right]_{-\infty}^{\infty}$ which is assumed to be finite by choosing $\theta_{0}$ appropriately. Eqs.(5.15) and (5.16) describe the evolution of the amplitude and velocity of the soliton respectively. It should be noted that when the inhomogeneity is absent the amplitude and velocity of the soliton do not change as time passes on.

To understand the nature of evolution of the amplitude and velocity of the soliton, we solve the set of coupled eqautions (5.15) and (5.16). On differentiating Eq.(5.15) with respect to T once and using Eq.(5.16) in the resultant equation and after rescaling T suitably, we obtain

$$
\eta_{T T}-(\eta-2) \eta^{2}=0
$$

Integrating Eq.(5.17) once, we get

$$
\left(\frac{d \eta}{d T}\right)^{2}-\frac{1}{2} \eta^{4}+\frac{4}{3} \eta^{3}=C_{0}
$$

where $C_{0}$ is the constant of integration. The left-hand side of (5.18) corresponds to the energy of the soliton which oscillates under the quartic potential. Now, we analyse Eq.(5.18) in two different cases namely when $C_{0}=0$ and $C_{0} \neq 0$ separately.

Case (i): $C_{0}=0$

Choosing the integration constant $C_{0}=0$, Eq.(5.18) can be easily integrated to give

$$
\eta=\frac{8}{3}\left[1-\frac{4}{9}\left(T+C_{1}\right)^{2}\right]^{-1}
$$

where $C_{1}$ is the second integration constant. $C_{1}$ can be evaluated by assuming that the initial amplitude of the soliton $\eta(0)$ as $\eta_{0}$. Now, after finding $C_{1}$ and transforming $T \rightarrow\left(T-\frac{1}{2}\right)$, Eq.(5.19) can be written as

$$
\eta=-\frac{6}{Q}
$$

where

$$
Q=\left[\left(T-\frac{1}{2}\right)^{2}+3\left(1-\frac{8}{3 \eta_{0}}\right)^{\frac{1}{2}}\left(T-\frac{1}{2}\right)-\frac{6}{\eta_{0}}\right] .
$$

Knowing $\eta$, the velocity $\xi$ of the soliton can be straightaway calculated by substituting Eq.(5.20) in the expression $\xi=\frac{\eta_{T}}{K(\eta-2)}$. Thus, the velocity of the soliton can be expressed as

$$
\xi=\frac{3}{K Q(Q+3)}\left[1-3\left(1-\frac{8}{3 \eta_{0}}\right)^{\frac{1}{2}}-2 T\right] .
$$

In Fig.1 and Fig.2, we have plotted the amplitude $(\eta)$ and velocity $(\xi)$ of the soliton as given in Eqs. (5.20) and (5.21) by assuming that the soliton is initially $(T=0)$ at rest $(\xi=0)$ and has an initial amplitude of $\eta_{0}=3.0$. As time passes, the amplitude and velocity of the soliton slowly increase and reach a maximum value. At this point the soliton suddenly flips and starts moving in the opposite direction and slowly dies out(damps) due to the inhomogeneity along the chain. As the velocity of the soliton is found to be inversely proportional to the inhomogeneity, in highly inhomogeneous magnetic media, the velocity of the soliton decreases very rapidly and also the 


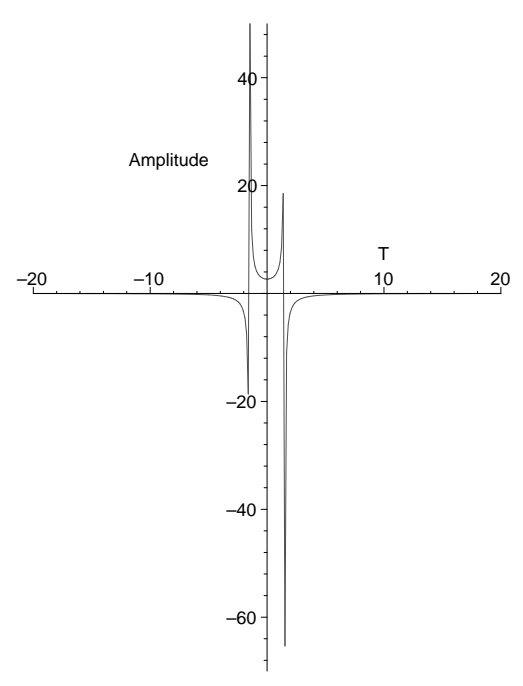

FIG. 1: Evolution of amplitude $(\eta)$ of the soliton when $C_{0}=0$ (Eq.(5.20)) under quadratic inhomogeneity when the initial amplitude is $\eta_{0}=3.0$.

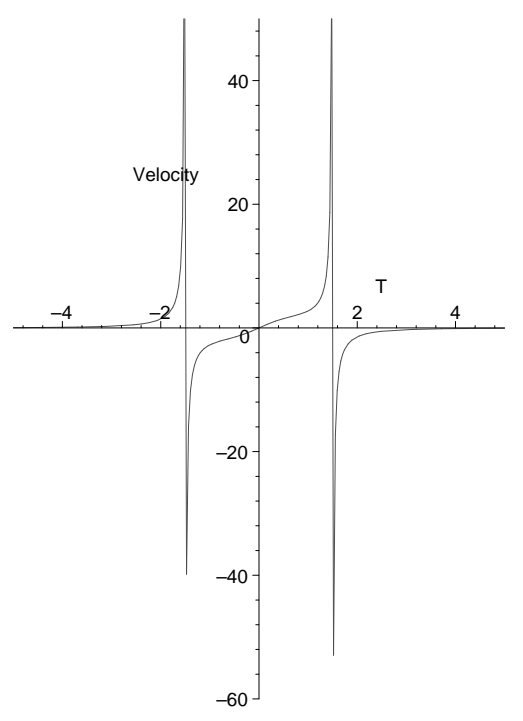

FIG. 2: Evolution of velocity $(\xi)$ of the soliton when $C_{0}=0$ (Eq.(5.21)) under quadratic inhomogeneity when the initial amplitude is $\eta_{0}=3.0$.

soliton dies out very quickly. It should be noted that if the soliton does not flip when it moves with very high speed it may explode suddenly.

Case (ii): $C_{0} \neq 0$

When $C_{0} \neq 0$, Eq.(5.18) can be integrated to give $\eta$ and hence also $\xi$ which can be written in terms of Jacobian elliptic functions [26]. For example (when $C_{0}$ is chosen as $\frac{7}{81}$ for convenient plotting 


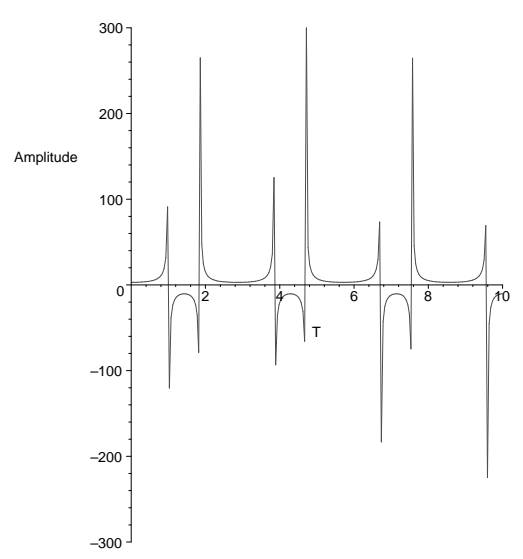

FIG. 3: Evolution of amplitude $(\eta)$ of the soliton when $C_{0}=\frac{7}{81}$ (Eq.(5.22)) under quadratic inhomogeneity when the initial amplitude is $\eta_{0}=3.0$.

of $\eta$ and $\xi) \eta$ can be explicitly written in the form

$$
\eta(T)=\frac{\left(\epsilon_{-}-C_{2} \epsilon_{+}\right)-\epsilon_{+} \operatorname{cn}\left(\frac{T}{g}\right)}{\left(\delta_{-}-C_{2} \delta_{+}\right)-\delta_{+} \operatorname{cn}\left(\frac{T}{g}\right)}
$$

where $\epsilon_{ \pm}=\eta_{2} a_{2} \pm \eta_{1} b_{2}, \quad \delta_{ \pm}=a_{2} \pm b_{2}, \quad C_{2}=\frac{2\left(\eta_{1}-\eta_{0}\right) b_{2}}{\eta_{0} \delta_{+}-\epsilon_{+}}, \quad g=\frac{1}{\sqrt{a_{2} b_{2}}}, \quad a_{1}^{2}=\frac{-\left(\eta_{3}-\eta_{3}^{*}\right)^{2}}{4}, \quad b_{1}=$ $\frac{\left(\eta_{3}+\eta_{3}^{*}\right)}{2}, \quad a_{2}^{2}=\left(\eta_{1}-b_{1}\right)^{2}+a_{1}^{2}, \quad b_{2}^{2}=\left(\eta_{2}-b_{1}\right)^{2}+a_{1}^{2}$. Here $\eta_{0}=\eta(0)$ as before and $\eta_{1}$ and $\eta_{2}$ are the two real roots of the polynomial $\eta^{4}-\frac{8}{3} \eta^{3}+\frac{7}{81}=0$ and $\eta_{3}$ is the complex root of the same. Using $\eta, \xi$ can be written as

$$
\xi=\frac{\left(\epsilon_{+} \delta_{-}-\delta_{+} \epsilon_{-}\right) \operatorname{sn}\left(\frac{T}{g}\right) d n\left(\frac{T}{g}\right)}{K g\left\{\left[\left(\delta_{-}-C_{2} \delta_{+}\right)-\delta_{+} \operatorname{cn}\left(\frac{T}{g}\right)\right]\left[\left(\epsilon_{-}-C_{2} \epsilon_{+}\right)-\epsilon_{+} c n\left(\frac{T}{g}\right)\right]-2\left[\left(\delta_{-}-C_{2} \delta_{+}\right)-\delta_{+} \operatorname{cn}\left(\frac{T}{g}\right)\right]^{2}\right\}},
$$

where $\operatorname{sn}\left(\frac{T}{g}\right), \operatorname{cn}\left(\frac{T}{g}\right)$ and $d n\left(\frac{T}{g}\right)$ are Jacobian elliptic functions.

In Figs.(3) and (4), we have plotted the amplitude $(\eta)$ and velocity $(\xi)$ of the soliton from Eqs.(5.22) and (5.23). In this case also, the amplitude of the soliton grows rapidly and suddenly flips. However, unlike the previous case this occurs periodically. Since $\eta$ is expressed in terms of Jacobian elliptic functions, in fact, it happens doubly periodically. As in the previous case, here also the velocity of the soliton grows rapidly and when it is at its highest speed and when it flips it suddenly moves in the opposite direction. Once again the velocity increases and the soliton turns back in the next flip. Interestingly, unlike the previous case here the soliton flip and its turn around happen doubly periodically. From Eq.(5.18) one can observe that $C_{0}$ represents the energy of the soliton and the soliton now oscillates doubly periodically under the potential $\left(\frac{-\eta^{4}}{2}+\frac{4}{3} \eta^{3}\right)$ (forced oscillations) in the inhomogeneous magnetic medium. Thus $C_{0}$ acts as a source of energy for the soliton to flip doubly periodically without dying and of course dies out when $C_{0}=0$. 


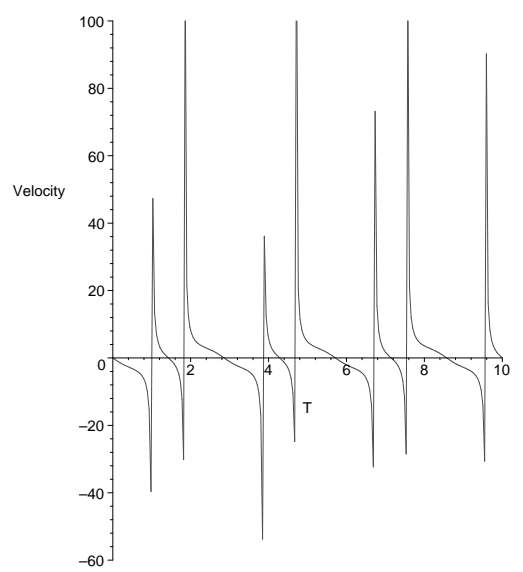

FIG. 4: Evolution of velocity $(\xi)$ of the soliton when $C_{0}=\frac{7}{81}$ (Eq.(5.23)) under quadratic inhomogeneity when the initial amplitude is $\eta_{0}=3.0$.

\section{Perturbed Solution}

The perturbed solution of Eq.(5.3) can be constructed by solving Eqs.(5.12) for $\phi_{1}$ and $\psi_{1}$ using the form of the inhomogeneity as $h_{1}=B\left(\theta-\theta_{0}\right)^{2}+C\left(\theta-\theta_{0}\right)+D$. The homogeneous part of Eq.(5.12a) admits the following two particular solutions.

$$
\begin{aligned}
\phi_{11}= & \operatorname{sech} \eta\left(\theta-\theta_{0}\right) \tanh \eta\left(\theta-\theta_{0}\right), \\
\phi_{12}= & -\frac{1}{\eta}\left[\operatorname{sech} \eta\left(\theta-\theta_{0}\right)-\frac{3}{2} \eta\left(\theta-\theta_{0}\right) \operatorname{sech} \eta\left(\theta-\theta_{0}\right) \tanh \eta\left(\theta-\theta_{0}\right)\right. \\
& \left.-\frac{1}{2} \tanh \eta\left(\theta-\theta_{0}\right) \sinh \eta\left(\theta-\theta_{0}\right)\right] .
\end{aligned}
$$

Knowing two particular solutions, the general solution can then be written in the form

$$
\phi_{1}=\delta_{1} \phi_{11}+\delta_{2} \phi_{12}-\phi_{11} \int_{-\infty}^{\theta} \phi_{12} \Re F_{1} d \theta^{\prime}+\phi_{12} \int_{-\infty}^{\theta} \phi_{11} \Re F_{1} d \theta^{\prime},
$$

where $\delta_{1}$ and $\delta_{2}$ are arbitrary constants of integration and $\Re F_{1}$ is as given in Eq.(5.12c). Substituting $\phi_{11}, \phi_{12}$ and $\Re F_{1}$ in (5.26) and evaluating the integrals and after lengthy calculations we 
obtain the solution as

$$
\begin{aligned}
\phi_{1}= & {\left[-\frac{\delta_{2}}{\eta}+\frac{15 B}{2 \eta^{2}}-\frac{9}{4 \eta}\left(\xi \theta_{0 T}+\sigma_{0 T}+2 B\right)-\frac{1}{2} \Delta\left[5 B\left(\theta-\theta_{0}\right)^{2}-C\left(\theta-\theta_{0}\right)\right.\right.} \\
& \left.\left.+\frac{9}{2 \eta} D\right]-\frac{K}{\eta}\left(\theta-\theta_{0}\right)+\left[\frac{5 B}{\eta^{2}}+\frac{D \Delta}{\eta^{2}}\right] \ln \cosh \eta\left(\theta-\theta_{0}\right)\right] \operatorname{sech} \eta\left(\theta-\theta_{0}\right) \\
& +\left[\frac{-15 B}{2 \eta^{2}}+\frac{7}{4 \eta}\left(\xi \theta_{0 T}+\sigma_{0 T}+2 B\right)+\frac{1}{4} \Delta\left[15 B\left(\theta-\theta_{0}\right)^{2}+7 D\right]-\frac{4 B}{\eta^{2}}\right. \\
& \left.X \ln \cosh \eta\left(\theta-\theta_{0}\right)\right] \operatorname{sech}{ }^{3} \eta\left(\theta-\theta_{0}\right)+\left[\delta_{1}+\frac{3 \delta_{2}}{2} \eta\left(\theta-\theta_{0}\right)+\frac{3}{4}\left(\theta-\theta_{0}\right)\right. \\
& X\left(\xi \theta_{0 T}+\sigma_{0 T}+2 B\right)+\frac{1}{2} \Delta\left(-\frac{3 K}{\eta^{2}}+5 B \eta^{2}\left(\theta-\theta_{0}\right)^{2}-\left[C \eta^{2}-\frac{5 D}{2} \eta\right.\right. \\
& \left.\left.\left.+\frac{6 B}{\eta}\left(\theta-\theta_{0}\right)\right]\right)\right] \operatorname{sech} \eta\left(\theta-\theta_{0}\right) \tanh \eta\left(\theta-\theta_{0}\right)-\left[\frac{3}{2 \eta} B \Delta\left(\theta-\theta_{0}\right)\right] \operatorname{sech} \eta\left(\theta-\theta_{0}\right) \\
& X \tanh \eta\left(\theta-\theta_{0}\right) \ln \cosh \eta\left(\theta-\theta_{0}\right)+\left[\frac{1}{4 \eta^{2}}\left[-2 K \Delta-9 \eta^{2}-\xi^{2}\right)\left(2 B\left(\theta-\theta_{0}\right)-2 C\right)\right] \\
& X \sinh \eta\left(\theta-\theta_{0}\right)-\left[\frac{B}{2 \eta^{2}} \Delta \tanh \eta\left(\theta-\theta_{0}\right)\right] \sinh \eta\left(\theta-\theta_{0}\right) \ln \cosh \eta\left(\theta-\theta_{0}\right) \\
& +\left[\frac{\delta_{2}}{2 \eta}+\frac{1}{4 \eta}\left(\xi \theta_{0 T}+\sigma_{0 T}+2 B+D \Delta\right)\right] \tanh \eta\left(\theta-\theta_{0}\right) \sinh \eta\left(\theta-\theta_{0}\right) \\
& -\frac{3 B}{\eta^{2}} \Delta \sum_{k=1}^{\infty} \frac{2^{2 k}\left(2^{2 k}-1\right) B_{2 k}\left(\eta\left(\theta-\theta_{0}\right)\right)^{2 k+1}}{(2 k+1) 2 k !} \operatorname{sech} \eta\left(\theta-\theta_{0}\right) \tanh \eta\left(\theta-\theta_{0}\right),
\end{aligned}
$$

where $\Delta=\left(\eta^{2}-\xi^{2}\right)$ and $B_{2 k}$ is the Bernoulli number. Before writing the final form of $\phi_{1}$, we remove the secular terms which make the solution unbounded by choosing the arbitrary constant $\delta_{2}$ as

$$
\delta_{2}=\frac{-1}{2}\left(\xi \theta_{0 T}+\sigma_{0 T}+2 B+D \Delta\right) .
$$

Using the boundary conditions $\left.\phi_{1}(0)\right|_{\theta_{0}=0}=\left.\phi_{1 \theta}(0)\right|_{\theta_{0}=0}=0$, we obtain

$$
\delta_{1}=\frac{3}{2 \eta^{2}} K \Delta
$$

and once again the same value of $\delta_{2}$ as given in Eq.(5.28). Using these results in the solution given in Eq.(5.26) the final form of the general solution $\phi_{1}$ can be written as

$$
\begin{aligned}
\phi_{1}= & {\left[\frac{3}{4}\left(\xi \theta_{0 T}+\sigma_{0 T}+2 B\right)\left(\left(\theta-\theta_{0}\right)-\frac{3}{\eta}\right)+D \Delta\left(\theta-\theta_{0}\right)+\frac{15 B}{2 \eta^{2}}-\frac{1}{2} \Delta\right.} \\
& X\left[5 B\left(\theta-\theta_{0}\right)^{2}-C\left(\theta-\theta_{0}\right)+\frac{9}{2 \eta} D-\frac{1}{\eta} K\left(\theta-\theta_{0}\right)\right]+\left(\frac{5 B}{\eta^{2}}+\frac{D \Delta}{\eta^{2}}\right) \\
& \left.X \ln \cosh \eta\left(\theta-\theta_{0}\right)\right] \operatorname{sech} \eta\left(\theta-\theta_{0}\right)+\left[\frac{-15 B}{2 \eta^{2}}+\frac{7}{4 \eta}\left(\xi \theta_{0 T}+\sigma_{0 T}+2 B\right)+\frac{1}{4} \Delta\right. \\
& \left.X\left[15 B\left(\theta-\theta_{0}\right)^{2}+7 D\right]-\frac{4 B}{\eta^{2}} \ln \cosh \eta\left(\theta-\theta_{0}\right)\right] \operatorname{sech}^{3} \eta\left(\theta-\theta_{0}\right)+\left[\frac{1}{2} \Delta\left(\theta-\theta_{0}\right)\right. \\
& \left.X\left(5 B \eta^{2}\left(\theta-\theta_{0}\right)-C \eta^{2}+\frac{11 D}{2} \eta-\frac{6 B}{\eta}-\frac{3 B}{\eta} \ln \cosh \eta\left(\theta-\theta_{0}\right)\right)\right] \\
& X \operatorname{sech} \eta\left(\theta-\theta_{0}\right) \tanh \eta\left(\theta-\theta_{0}\right) .
\end{aligned}
$$


Following the same procedure $\psi_{1}$ can also be obtained by solving Eq.(5.12b). The solutions of the homogeneous part of Eq.(5.12b) read

$$
\begin{aligned}
& \psi_{11}=\operatorname{sech} \eta\left(\theta-\theta_{0}\right) \\
& \psi_{12}=\frac{1}{2 \eta}\left[\eta\left(\theta-\theta_{0}\right) \operatorname{sech} \eta\left(\theta-\theta_{0}\right)+\sinh \eta\left(\theta-\theta_{0}\right)\right] .
\end{aligned}
$$

As before, the general solution of Eq.(5.12b) can be written in the form

$$
\psi_{1}=\delta_{3} \psi_{11}+\delta_{4} \psi_{12}-\psi_{11} \int_{-\infty}^{\theta} \psi_{12} \Im F_{1} d \theta^{\prime}+\psi_{12} \int_{-\infty}^{\theta} \psi_{11} \Im F_{1} d \theta^{\prime}
$$

where $\delta_{3}$ and $\delta_{4}$ are arbitrary constants. Substituting the solutions $\psi_{11}$ and $\psi_{12}$ from Eqs.(5.31) and (5.32) in Eq.(5.33) and putting $\Im F_{1}$ after evaluating the value of it for the above quadratic inhomogeneity and after finding out the value of the integrals in Eq.(5.33), the general solution for $\psi_{1}$ after making lengthy calculations can be written as

$$
\begin{aligned}
\psi_{1}= & {\left[\delta_{3}+\frac{\left(\theta-\theta_{0}\right)}{2}\left[\frac{\delta_{4}}{2}+\wp\left(\theta-\theta_{0}\right)+\frac{\eta}{2}\left(\theta-\theta_{0}\right)_{T}+\frac{1}{\eta}\left[\xi \eta^{2}\left(\theta-\theta_{0}\right)^{2}+\frac{C}{2}\left(\theta-\theta_{0}\right)\right.\right.\right.} \\
& \left.\left.\left.+D-D \xi \eta^{2}\right]\right]\right] \operatorname{sech} \eta\left(\theta-\theta_{0}\right)+\left[\frac { ( \theta - \theta _ { 0 } ) } { 2 } \left[-\wp\left(\theta-\theta_{0}\right)\right.\right. \\
& \left.-\frac{\eta}{2}\left(\theta-\theta_{0}\right)_{T}-\xi \eta\left[B\left(\theta-\theta_{0}\right)^{2}-C\left(\theta-\theta_{0}\right)+D\right]\right]+\frac{1}{2} \eta\left(\theta-\theta_{0}\right)_{T}+\xi \eta D-\frac{\xi B}{\eta^{2}} \\
& \left.\sum_{k=1}^{\infty} \frac{2^{2 k}\left(2^{2 k}-1\right) B_{2 k}\left(\eta\left(\theta-\theta_{0}\right)\right)^{2 k+1}}{(2 k+1) 2 k !}\right] \operatorname{sech}^{3} \eta\left(\theta-\theta_{0}\right)+\left[\frac { 1 } { 2 } \left[\frac{\wp}{\eta}\left(\theta-\theta_{0}\right)\right.\right. \\
& \left.\left.+\frac{1}{2}\left(\theta-\theta_{0}\right)_{T}-\frac{11}{2} B \xi\left(\theta-\theta_{0}\right)^{2}+D \xi\right]\right] \operatorname{sech} \eta\left(\theta-\theta_{0}\right) \tanh \eta\left(\theta-\theta_{0}\right)+\left[\frac{1}{\eta} \xi B\left(\theta-\theta_{0}\right)\right] \\
& \operatorname{sech}\left(\theta-\theta_{0}\right) \ln \cosh \eta\left(\theta-\theta_{0}\right)+\left[\frac{6}{\eta} B \xi\left(\theta-\theta_{0}\right) \sinh \eta\left(\theta-\theta_{0}\right)\right] \ln \cosh \eta\left(\theta-\theta_{0}\right) \\
& +\frac{1}{2 \eta^{2}}\left[\wp-2 B \xi \eta\left(\theta-\theta_{0}\right)-C \xi \eta\right] \sinh \eta\left(\theta-\theta_{0}\right) \tanh \eta\left(\theta-\theta_{0}\right) \\
& +\left[\frac{1}{4 \eta}\left[2 \delta_{4}-\eta\left(\theta-\theta_{0}\right)_{T}-2 D \eta \xi\right]\right] \sinh \eta\left(\theta-\theta_{0}\right),
\end{aligned}
$$

where $\wp=K \xi(\eta-2)$. The secular terms can be removed by choosing

$$
\delta_{4}=\frac{\eta}{2}\left(\theta-\theta_{0}\right)_{T}+2 D \xi .
$$

The boundary conditions $\left.\psi_{1}(0)\right|_{\theta_{0}=0}=\left.\psi_{1 \theta}(0)\right|_{\theta_{0}=0}=0$, give

$$
\begin{aligned}
\delta_{3} & =D \xi \eta \\
\delta_{4} & =-D \xi \eta .
\end{aligned}
$$


Using these results, the final form of the general solution $\psi_{1}$ is written as

$$
\begin{aligned}
\psi_{1}= & {\left[D \xi \eta+\left[-\frac{1}{2} D \xi \eta+\wp\left(\theta-\theta_{0}\right)+\frac{1}{\eta}\left[\xi \eta^{2}\left(\theta-\theta_{0}\right)^{2}+\frac{C}{2}\left(\theta-\theta_{0}\right)+D\right.\right.\right.} \\
& \left.\left.\left.-3 D \xi \eta^{2}\right]\right] \frac{\left(\theta-\theta_{0}\right)}{2}\right] \operatorname{sech} \eta\left(\theta-\theta_{0}\right)+\left[\left[-\wp\left(\theta-\theta_{0}\right)-\xi \eta\left[B\left(\theta-\theta_{0}\right)^{2}\right.\right.\right. \\
& \left.\left.\left.-C\left(\theta-\theta_{0}\right)+D\right] \frac{\left(\theta-\theta_{0}\right)}{2}\right]-2 D \xi \eta^{2}+\xi \eta D-\frac{\xi B}{\eta^{2}}\right] \operatorname{sech}^{3} \eta\left(\theta-\theta_{0}\right) \\
+ & {\left[\frac{1}{2}\left[\frac{1}{\eta} \wp\left(\theta-\theta_{0}\right)-2 D \xi \eta-\frac{11}{2} \xi B\left(\theta-\theta_{0}\right)^{2}+\xi D\right]\right] \operatorname{sech} \eta\left(\theta-\theta_{0}\right) \tanh \eta\left(\theta-\theta_{0}\right) } \\
+ & {\left[\frac{1}{\eta} B \xi\left(\theta-\theta_{0}\right)\right] \operatorname{sech} \eta\left(\theta-\theta_{0}\right) \ln \cosh \eta\left(\theta-\theta_{0}\right) . }
\end{aligned}
$$

Thus, the first order perturbed soliton solution $\hat{q}_{1}$ is obtained as

$$
\begin{aligned}
& \hat{q}_{1}=\left[\frac{3}{4}\left(\xi \theta_{0 T}+\sigma_{0 T}+2 B\right)\left(\left(\theta-\theta_{0}\right)-\frac{3}{\eta}\right)+D \Delta\left(\theta-\theta_{0}\right)+\frac{15 B}{2 \eta^{2}}-\frac{1}{2} \Delta\left[5 B\left(\theta-\theta_{0}\right)^{2}\right.\right. \\
& \left.\left.-C\left(\theta-\theta_{0}\right)+\frac{9}{2 \eta} D-\frac{1}{\eta} K\left(\theta-\theta_{0}\right)\right]+\left(\frac{5 B}{\eta^{2}}+\frac{D \Delta}{\eta^{2}}\right) \ln \cosh \eta\left(\theta-\theta_{0}\right)\right] \\
& X \operatorname{sech} \eta\left(\theta-\theta_{0}\right)+\left[\frac{-15 B}{2 \eta^{2}}+\frac{7}{4 \eta}\left(\xi \theta_{0 T}+\sigma_{0 T}+2 B\right)+\frac{1}{4} \Delta\left[15 B\left(\theta-\theta_{0}\right)^{2}+7 D\right]\right. \\
& \left.-\frac{4 B}{\eta^{2}} \ln \cosh \eta\left(\theta-\theta_{0}\right)\right] \operatorname{sech}^{3} \eta\left(\theta-\theta_{0}\right)+\left[\frac { 1 } { 2 } \Delta ( \theta - \theta _ { 0 } ) \left(5 B \eta^{2}\left(\theta-\theta_{0}\right)-C \eta^{2}\right.\right. \\
& \left.\left.+\frac{11 D}{2} \eta-\frac{6 B}{\eta}-\frac{3 B}{\eta} \ln \cosh \eta\left(\theta-\theta_{0}\right)\right)\right] \operatorname{sech} \eta\left(\theta-\theta_{0}\right) \tanh \eta\left(\theta-\theta_{0}\right) \\
& +i\left[\left[D \xi \eta+\left[-\frac{1}{2} D \xi \eta+\wp\left(\theta-\theta_{0}\right)+\frac{1}{\eta}\left[\xi \eta^{2}\left(\theta-\theta_{0}\right)^{2}+\frac{C}{2}\left(\theta-\theta_{0}\right)+D\right.\right.\right.\right. \\
& \left.\left.\left.-3 D \xi \eta^{2}\right]\right] \frac{\left(\theta-\theta_{0}\right)}{2}\right] \operatorname{sech} \eta\left(\theta-\theta_{0}\right)+\left[\left[-\wp\left(\theta-\theta_{0}\right)-\xi \eta\left[B\left(\theta-\theta_{0}\right)^{2}\right.\right.\right. \\
& \left.\left.\left.-C\left(\theta-\theta_{0}\right)+D\right] \frac{\left(\theta-\theta_{0}\right)}{2}\right]-2 D \xi \eta^{2}+\xi \eta D-\frac{\xi B}{\eta^{2}}\right] \operatorname{sech}^{3} \eta\left(\theta-\theta_{0}\right) \\
& +\left[\frac{1}{2}\left[\frac{1}{\eta} \wp\left(\theta-\theta_{0}\right)-2 D \xi \eta-\frac{11}{2} \xi B\left(\theta-\theta_{0}\right)^{2}+\xi D\right]\right] \operatorname{sech} \eta\left(\theta-\theta_{0}\right) \tanh \eta\left(\theta-\theta_{0}\right) \\
& \left.+\left[\frac{1}{\eta} B \xi\left(\theta-\theta_{0}\right)\right] \operatorname{sech} \eta\left(\theta-\theta_{0}\right) \ln \cosh \eta\left(\theta-\theta_{0}\right)\right] \text {. }
\end{aligned}
$$

Knowing $\hat{q}_{1}$ the general perturbed solution can be written down. Using these results the spin components can be constructed using the known differential geometric considerations.

\section{CONCLUSIONS}

In this paper, we have investigated the integrability of the classical one dimensional Heisenberg inhomogeneous ferromagnetic spin chain and also the effect of inhomogeneity on the soliton of the associated completely integrable spin model. The spin dynamics of the system has been studied by mapping the spin chain onto a moving space curve. The resultant invariant equations are expressed in the form of an inhomogeneous higher order generalized NLS equation. By carrying out Painlevé singularity structure analysis we found that the system is in general not integrable and becomes 
integrable for linear inhomogeneity and for specific choice of biquadratic exchange interaction. After mentioning the Lax pair and soliton solutions of the integrable equation, we investigate the effect of nonlinear(quadratic) inhomogeneity on the soliton of an underlying integrable model of the inhomogeneous spin system at lower order. For this, we carried out a mulitple scale perturbation analysis and constructed the perturbed soliton. More interestingly we found that under the influence of the nonlinear inhomogeneity, the amplitude and velocity of the soliton undergo curious changes. As time passes on the amplitude and velocity of the soliton increase and when it moves with very high speed it flips suddenly and turns back and slows down, once again flips and this happens doubly periodically.

\section{APPENDIX A: EVALUATING THE SECULARITY CONDITIONS}

Using the value of $\Re F_{1}$ form Eq.(5.12c) in the secularity condition

$$
\begin{aligned}
& \int_{-\infty}^{\infty} \hat{q}_{0 \theta} \Re F_{1} d \theta \equiv \int_{-\infty}^{\infty} \hat{q}_{0 \theta}\left[\hat { q } _ { 0 } \left(h_{1 \theta \theta}+2 h_{1}\left|\hat{q}_{0}\right|^{2}-h_{1} \xi^{2}+\xi \theta_{0 T}+\sigma_{0 T}\right.\right. \\
& \left.-\xi_{T}\left(\theta-\theta_{0}\right)+2 \int_{\infty}^{\theta} h_{1 \theta^{\prime}}\left|q_{0}\right|^{2} d \theta^{\prime}+2 h_{1 \theta} \hat{q}_{0 \theta}+h_{1} q_{0 \theta \theta}\right]=0
\end{aligned}
$$

where $h_{1}(\theta)=B\left(\theta-\theta_{0}\right)^{2}+C\left(\theta-\theta_{0}\right)+D$ and using $\hat{q}_{0}$ and their derivatives in the above equation, we obtain

$$
\begin{aligned}
& \int_{-\infty}^{\infty}\left[\xi_{T}\left(\theta-\theta_{0}\right)-\xi \theta_{0 T}-\sigma_{0 T}-\left(\eta^{2}-\xi^{2}\right)\left(B\left(\theta-\theta_{0}\right)+D\right)-2 B\right. \\
& \left.-2 \eta^{2} \int_{-\infty}^{\theta^{\prime}}\left(2 B\left(\theta-\theta_{0}\right)+C\right) \operatorname{sech}^{2} \eta\left(\theta^{\prime}-\theta_{0}^{\prime}\right) d \theta^{\prime}\right\} \operatorname{sech}^{2} \eta\left(\theta-\theta_{0}\right) \tanh \eta\left(\theta-\theta_{0}\right) \\
& +2 \eta\left(2 B\left(\theta-\theta_{0}\right)+C\right) \operatorname{sech}^{2} \eta\left(\theta-\theta_{0}\right)-2 \eta\left(2 B\left(\theta-\theta_{0}\right)+C\right) \\
& \left.X \operatorname{sech}^{4} \eta\left(\theta-\theta_{0}\right)\right] d \theta=0 .
\end{aligned}
$$

Evaluating the above integrals, Eq.(A2) gives

$$
\xi_{T}=2 K\left(\eta^{2}-\xi^{2}\right) .
$$

Similarly, substituting the value of $\Im F_{1}$ from Eq.(5.12d) we obtain

$$
\int_{-\infty}^{\infty} \hat{q}_{0} \Im F_{1} d \theta \equiv \hat{q}_{0}\left[\hat{q}_{0 T}+2 \xi h_{1 \theta}+2 \xi h_{1} \hat{q}_{0 \theta}\right] d \theta=0 .
$$

Making use of $\hat{q}_{0}$ we get

$$
\begin{aligned}
& \int_{-\infty}^{\infty}\left[\left[\eta_{T}+2 \eta \xi h_{1 \theta}\right] \operatorname{sech}^{2} \eta\left(\theta-\theta_{0}\right)-\left[\eta\left(\eta\left(\theta-\theta_{0}\right)\right)_{T}+2 \eta^{2} \xi h_{1}\right]\right. \\
& \left.\operatorname{sech}^{2} \eta\left(\theta-\theta_{0}\right) \tanh \eta\left(\theta-\theta_{0}\right)\right] d \theta=0 .
\end{aligned}
$$

Evaluating Eq.(A4) we obtain

$$
\eta_{T}=2 K \xi(\eta-2)
$$




\section{ACKNOWLEDGMENTS}

The work of M.D forms part of a major research project sponsored by the DST, Govt. of India. The major part of the work was carried out at the Abdus Salam International Centre for Theoretical Physics, Trieste, Italy, within the framework of the Associateship Scheme. The authors acknowledge the support received from the Abdus Salam ICTP.

\section{References}

1 L. Landau, and E. Lifshitz, Phys. Z. Sowjetunion 8, 153 (1935).

2 M. Lakshmanan, Th.W. Ruijgrok, and C. J. Thompson, Physica A84, 577 (1976).

3 M. Lakshmanan, Phys. Lett. A61, 53 (1977).

4 V. E. Zakharov, and L.A. Takhtajan, Theor. Math. Phys. 38, 7 (1979).

5 M. Lakshmanan, K. Porsezian, and M. Daniel, Phys. Lett. A133, 483 (1998).

6 K. Porsezian, M. Daniel, and M. Lakshmanan, J. Math. Phys. 33, 1807 (1992).

7 M. Daniel, K. Porsezian and M. Lakshmanan J. Math. Phys. 35, 6498 (1994).

8 M. Daniel, and R. Amuda, Phys. Rev. B53, R2930 (1996).

9 M. Daniel, L. Kavitha, and R. Amuda, Phys. Rev. B59, 13774 (1999).

10 M. Daniel, and L. Kavitha, Phys. Rev. B63, 172302 (2001).

11 M . Lakshmanan, and R. K. Bullough, Phys. Lett. A80, 287 (1980).

12 M . Lakshmanan, and S. Ganesan, Physica A132, 117 (1985).

13 V. E. Zakharov, Sov. Phys. JETP 72, 908 (1972).

14 J. J. Stoker, Differential Geometry (Wiley, New York, 1969).

15 K. Porsezian, M. Daniel, and R. Bharathikannan, Phys. Lett. A156, 206 (1991).

16 M. J. Ablowitz, A. Ramani, and H.Segur, J. Math. Phys. 21, 715 (1980).

17 J. Weiss, M. Tabor, and G. Carnevale, J. Math. Phys. 24, 522 (1983).

18 M.J. Ablowitz, D.J. Kaup, A.C. Newell, and H. Segur, Stud. Appl. Math. 53, 249 (1974).

19 M. J. Ablowitz, and H.Segur, Solitons and Inverse Scattering Transform (SIAM, Philadelphia, PA, 1981).

20 R. M. Miura, Bäcklund Transformations, Lecture Notes in Mathematics (SpringerVerlag, Berlin, 1976). 
21 K. Konno, and M. Wadati, Prog. Theor. Phys. 53, 1652 (1975).

22 M. Daniel, and M. Lakshmanan, Physica A120, 125 (1983).

23 H. Fogedby, J. Phys. A13, 1467 (1980).

24 Y. Kodama, and M. J. Ablowitz, Stud. Appl. Math. 64, 225 (1981).

25 Y. Kodama, J. Stat. Phys. 39, 516 (1986).

26 P. F. Byrd and M. D. Friedman, Handbook of Elliptic Integrals for Engineers and Scientists (Springer-Verlag, Berlin, 1971). 